\title{
Tobacco smoking predicts depression and poorer quality of life in heart disease
}

Lesley Stafford ${ }^{1,2,6^{*}}$, Michael Berk $3,4,5,6$ and Henry J Jackson ${ }^{2}$

\begin{abstract}
Background: We report on the prospective association between smoking and depression and health-related quality of life (HRQOL) in patients with coronary artery disease (CAD).

Methods: Prospective study of 193 patients with assessment of depression occurring 3-, 6- and 9- months (T1, 2, and 3, respectively) following discharge from hospital for a cardiac event. HRQOL was assessed at T3. T1 depression was assessed by clinical interview; T2 and T3 depression was assessed by self-report. Smoking at time of cardiac event was assessed by self-report. Multivariate analyses controlled for known demographic, psychosocial and clinical correlates of depression.
\end{abstract}

Results: Smoking at the time of index cardiac event increased the likelihood of being diagnosed with Major Depressive Disorder (MDD) at T1 by 4.30 [95\% Cl, 1.12-16.46; $p<.05]$. The likelihood of receiving a diagnosis of minor depression, dysthymia or MDD as a combined group was increased by 8.03 [95\% Cl, 2.35-27.46; $p<.01]$. Smoking did not reliably predict depression at T2 or T3 and did not reliably predict persistent depression. Smoking increased the likelihood of being classified as depressed according to study criteria at least once during the study period by $5.19[95 \% \mathrm{Cl}, 1.51-17.82$; $p<.01]$. Smoking independently predicted worse mental HRQOL.

Conclusions: The findings support a role for smoking as an independent predictor of depression in CAD patients, particularly in the first 3 months post-cardiac event. The well-established imperative to encourage smoking cessation in these patients is augmented and the findings may add to the evidence for smoking cessation campaigns in the primary prevention of depression.

Keywords: Coronary artery disease, Depression, Smoking, Quality of life

\section{Background}

Tobacco smoking is a leading global cause of preventable morbidity and mortality [1]. Despite a steady recent decrease in smoking prevalence in the general population, smoking remains responsible for approximately $8 \%$ of the overall burden of disease and injury [2]. Although there is substantial recognition of the effect of smoking on physical morbidity, there is lesser appreciation of its connection to psychological morbidity, including depression. Smoking is disproportionately common among individuals with psychiatric disorders [3-6] and there is evidence that it is noxious to mental health [7-9]. Smoking also appears

\footnotetext{
* Correspondence: lesley.stafford@thewomens.org.au

'Centre for Women's Mental Health, Royal Women's Hospital, Parkville, Australia

${ }^{2}$ Melbourne School of Psychological Sciences, University of Melbourne, Melbourne, Australia

Full list of author information is available at the end of the article
}

to influence some of the pathophysiological pathways that are germane to depression [10].

A reciprocal relationship exists between depression and smoking such that depression is more common in smokers than non-smokers, and smoking is more common in depressed versus non-depressed individuals. Studies in the general population have found that tobacco smoking is a risk factor for depression [11,12]. A recent study showed that the presence of smoking doubled the risk of development of a de-novo episode of major depression in women followed up for 10 years [13].

Both depression [14-16] and smoking $[17,18]$ are risk factors for cardiac morbidity and mortality in patients with CAD; however, previous data on the association between tobacco smoking and depression in CAD patients are inconsistent. Some studies report no association [19-21] and others report that smoking is a risk factor

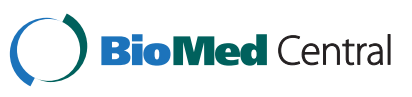


for depression in CAD [22,23]. We conducted an analysis of data from a longitudinal study of depression in CAD to investigate the prospective association between smoking and depression. Based on the weight of evidence, we hypothesized that smoking would be associated with increased risk for the subsequent onset of depression among recently hospitalized CAD patients when known correlates of depression were controlled $a$ priori. These correlates include clinical variables such as disease severity, body mass index (BMI), having undergone coronary artery bypass graft (CABG) surgery, diabetes, alcohol intake, functional disability, prior history of depression, Generalized Anxiety Disorder (GAD), neuroticism, and known socio-demographic factors such as gender, income, marital status and social support,). As a secondary outcome, we investigated the association between smoking and health-related quality of life (HRQOL). A deleterious impact of smoking on HRQOL has been reported in cross-sectional samples of the general population [24-26]. Only one prior longitudinal study has explicitly examined this association in CAD patients and showed that continued smoking after percutaneous transluminal coronary angioplasty (PTCA) significantly diminished the HRQOL benefits of this procedure [27]. We further hypothesized that smoking would be associated with worse HRQOL. The aims of the study were therefore to investigate the prospective association between smoking and depression and smoking and HRQOL in a cohort with cardiovascular disease.

\section{Methods}

\section{Sample}

Participants were recruited between May 2005 and March 2006 from a tertiary hospital in regional Australia that serves a catchment area shown to be representative of the broader Australian community [28]. All English-speaking, consenting patients who were hospitalized for PTCA, myocardial infarction (MI) or CABG during this time were eligible for participation. According to discharge diagnoses, 528 patients were treated for these presentations during the study period. Patients with different CAD presentations were regarded as a homogenous group based on evidence that the association between depression and CAD is found across all subgroups of CAD patients with similar prognostic effects for cardiac $[14,29]$ and HRQOL $[30,31]$ outcomes. Participants were recruited by postal invitation and follow-up phone-call 6 weeks post-discharge. Reasons for non-participation were death $(n=13)$, non-English speaking $(n=16)$, unable to consent $(n=9)$, medically unwell $(n=9)$, depression $(n=3)$ and no given reason $(n=249)$. Agreement to participate was obtained from 229 patients. The study received ethics approval from the Barwon Health Human Research and Ethics Committee and all participants gave written informed consent.

\section{Procedures}

Data were collected at 3- (T1), 6- (T2) and 9 months (T3) after hospital discharge. Baseline assessment was undertaken 3 months post-discharge, rather than during admission, as well as to avoid potential confounding of the effects of acute illness and stress associated with hospitalization with the assessment of predictor variables and to minimize the burden on participants. Other studies [22,23,32] have highlighted difficulties in reliably identifying patients in whom depression will emerge, persist or worsen only on the basis of level of depressive symptomatology present during hospitalization. Structured clinical interviews were administered telephonically at T1. Telephonic administration of structured clinical interviews has been found to be valid and reliable [33,34]. For analyses, participants were assigned to a diagnostic group "major depressive disorder" (MDD) or to a broader group "any depressive disorder" ('ADD'). The latter comprised, in addition to participants with MDD, participants with minor depression and dysthymia.

Participants were also mailed self-report questionnaires at T1, T2 and T3. T1 questionnaires assessed neuroticism, social support, and functional disability. The depression self-report measure was administered at T2 and T3. The structured clinical interview was used to establish a diagnosis while the self-report depression measure enabled tracking of depression change over time. HRQOL data were collected at T3. Relevant clinical and socio-demographic data were collected by self-report and from medical records at $\mathrm{T} 1$.

\section{Measures}

Clinical data, including discharge diagnosis, antidepressant therapy at discharge, disease severity and the presence of hypertension and diabetes, were obtained from medical records. Disease severity was measured with left ventricular ejection fraction (LVEF). BMI was computed based on self-reported height and weight $\left(\mathrm{kg} / \mathrm{m}^{2}\right)$ at the time of the index event. Data on commencement of antidepressant therapy after index event, attendance at cardiac rehabilitation, marital status, annual household income, prior history of depression, and tobacco use and alcohol consumption at the index event were gathered by self-report. Alcohol consumption was considered excessive if greater than the quantity recommended by the National Heart Foundation of Australia ( $\leq 7$ and 14 standard drinks per week for women and men, respectively) [35]. Low household income was categorized as $\leq$ $\$ 20000$ (Australian).

Clinical depression and GAD were assessed using the Mini International Neuropsychiatric Interview Version 5 (M.I.N.I.) [36,37], a diagnostic structured interview compatible with ICD-10 and DSM-IV criteria, and similar to the Structured Clinical Interview for DSM-IV Disorders 
(SCID) [38] in operation and principle. The M.I.N.I. has excellent psychometric properties and has been validated against the SCID-Patient Version [39] and the Composite International Diagnostic Interview (CIDI) [36,37,40,41]. M. I.N.I modules for depression, GAD and dysthymia were administered. Major depressive disorder (MDD) was diagnosed if participants fulfilled DSM-IV criteria of at least one core criterion (depressed mood or anhedonia) and at least four additional criteria within a 2-week duration. With the use of the same module, minor depression was diagnosed if participants fulfilled at least one core criterion and one to three additional criteria within a 2-week period.

Depressive symptomatology was measured with the 7item depression subscale of the Hospital Anxiety and Depression Scale (HADS) [42]. The HADS has wellestablished psychometric properties [43,44] and is widely used in studies with cardiac patients [45-47]. Possible scores range from 0 to 21 . Higher scores denote greater depressive symptomatology. Cronbach's alpha at T1, T2 and T3 were $.81, .82$, and .84, respectively. In accordance with previous data $[44,48-50]$, a score $\geq 8$ was considered to indicate depression.

Social support was assessed with the psychometricallyrobust [51-53] and widely-used [49,54-56] 12-item Multidimensional Scale of Perceived Social Support (MSPSS) [57]. This scale measures support from family, friends and a significant other. The total score ranges from 7 to 84 with higher scores indicating higher levels of support. Cronbach's alpha was .93.

Neuroticism was measured with a 10-item scale from the self-report version of the NEO PI-R [58], the IPIP-NEO [59]. Scores range from 10 to 50 with higher scores indicating greater neuroticism. The IPIP-NEO has been shown to be reliable [60] and valid [58,61] and its factor structure has been confirmed $[62,63]$. Cronbach's alpha was .88 .

HRQOL was measured with the Short Form-36 (SF-36) [64], an inventory that assesses eight domains of HRQOL: physical functioning, bodily pain, fatigue, role limitations due to physical health problems, emotional functioning, role limitations due to emotional problems, social functioning and general health perception. Aggregate summary scores for physical functioning (PCS) and mental functioning (MCS) were calculated according to the standard algorithm [64]. Higher scores denote better functioning. Psychometric evidence suggests that the SF-36 is the most reliable, valid, and sensitive generic measure of HRQOL for use in CAD patients [65]. Cronbach's alpha values for the eight subscales at $\mathrm{T} 1$ and $\mathrm{T} 3$ ranged from .80 to .92 , and .81 to .93 , respectively.

Functional disability was measured using the 10 items from the SF-36 that assess physical functioning. Items include activities such as walking one block and climbing a flight of stairs. In the original context these items assess limitations on physical activities. Here, the response format was altered to assess the severity of symptoms experienced when performing these physical tasks. The items are similar to measures like the Canadian Cardiovascular Society for angina severity [66] and the New York Heart Association classification criteria for the prescription of physical activity for cardiac patients [67]. Responses include $I$ do not experience symptoms, I experience mild symptoms, I experience severe symptoms, and I am not able to perform this activity. Scores range from 0 to 30 with lower scores indicating greater functional disability.

\section{Analysis}

Data were analysed using SPSS. All tests were two-tailed and $\alpha$ was .05 . Univariate analyses comprised Chi-square and independent sample $t$-tests, as appropriate. Multivariate analyses to predict the association between smoking and depression used direct logistic regression. All predictor variables were entered in one block. Regression coefficients, Wald statistics, odds ratios (ORs) and 95\% confidence intervals were calculated. Regression analyses for T1 comprised predicting MDD and 'ADD', as defined above. At T2 and T3, depression was defined by a HADS score $\geq 8$. Two additional independent variables were created. Participants were assigned a priori to a 'persistently depressed' group if they were classified as depressed at T1, T2 and T3. Participants were assigned to an 'ever depressed' group if they were classified as depressed at T1, T2 or T3. For these two variables, membership of the 'ADD' group constituted T1 depression. Multivariate analyses to predict physical and mental HRQOL at T3 were performed using standard regression.

\section{Results}

\section{Characteristics of the sample}

T1 questionnaires were completed by 193 of the 229 recruited patients $(84 \%$; or $37 \%$ of all patients treated during the study period included those deceased and ineligible). According to discharge diagnoses, these 193 participants were treated for MI $(\mathrm{n}=13,7 \%)$, CABG ( $\mathrm{n}=91,47 \%)$, PTCA $(\mathrm{n}=35,18 \%), \mathrm{MI}$ and CABG $(\mathrm{n}=21,11 \%)$, and MI and PTCA $(\mathrm{n}=35,18 \%)$. [To facilitate analysis, this variable was categorized into CABG vs MI/PTCA]. Compared to non-participants, participants were significantly more likely to be male $\left[\chi^{2}\right.$ $(1, N=528)=5.93, P=.02]$ and were, on average, younger $[t(526)=3.12, P<.01]$. The mean $(\mathrm{SD})$ age for participants versus non-participants was 64.14 (10.37) versus 67.31 (11.70), respectively. Twenty-eight participants did not return their questionnaires for an unspecified reason, 3 withdrew due to physical illness and 4 withdrew due to depression. There were no significant gender differences between those who completed T1 questionnaires and those who did not $\left[\chi^{2}(1, N=229)=.64\right.$, 
$P=.42]$, but non-respondents were, on average, significantly younger than respondents $[t(227)=-2.13, P<.03]$. The mean (SD) age for non-responders was 59.94 (13.22). At T2, 189 of the $193 \mathrm{~T} 1$ participants remained. By T3, 184 of the $193 \mathrm{~T} 1$ participants remained (95\%; or $35 \%$ of the original patient group (184 of 528)). One participant had formally withdrawn due to depressive illness and 8 participants did not return their questionnaires for unspecified reasons. There were no gender or age differences between participants who completed T2 and T3 questionnaires and those who did not. Baseline clinical, sociodemographic, and psychosocial data for the sample and for non-smokers versus smokers are described in Table 1.

\section{Association between smoking and depression}

Twenty-seven participants (14\%) were smokers at the time of index event. At T1, 35 (18\%) participants were diagnosed with MDD and 54 (28\%) with 'ADD'. Univariate analyses (Table 1) showed that compared to non-smokers, participants who were smokers at the time of the index cardiac event were significantly younger $[t(191)=3.73$, $P<.001]$; less likely to have undergone CABG $\left[\chi^{2}(1, N=\right.$ $193)=10.4, P=.001]$, less likely to attend cardiac rehabilitation $\left[\chi^{2}(1, N=187)=6.98, P=.02\right]$, perceived less social support $[t(191)=2.70, P<.01]$, reported higher levels of neuroticism $[t(31.72)=-2.79, P<.01]$ and were more likely to receive a diagnosis of $\operatorname{GAD}\left[\chi^{2}(1, N=193)=6.47\right.$, $P=.01]$, 'ADD' $\left[\chi^{2}(1, N=193)=23.32, P<.001\right]$ or MDD $\left[X^{2}(1, N=193)=14.64, P<.001\right]$ at T1 (i.e., 3 months later).

Logistic regression analyses to predict depression are shown in Table 2 (MDD, 'ADD', and T2 Depression) and Table 3 (T3 Depression, 'Persistently depressed', and 'Ever depressed'). Inspection of the ORs showed that smoking at the time of index cardiac event increased the likelihood of being diagnosed with MDD at T1 by 4.30 [95\% CI, 1.12-16.46; $P<.05]$, an OR second only to that associated with a diagnosis of GAD. In the analysis to predict 'ADD' at T1, smoking had an OR of 8.03 [95\% CI, 2.3527.46; $P<.01]$ and was the strongest independent predictor of outcome of all the available variables. At T2 and T3, 24 (13\%) and 29 (16\%) participants, respectively, reported a HADS score $\geq 8$. Smoking did not reliably predict a depression at T2 and T3. Fourteen participants (8\%) were assigned to the 'persistently depressed' group and 60 (33\%) were assigned to the 'ever depressed' group. Smoking was not reliably associated with classification as 'persistently depressed'. Smoking increased the

Table 1 Characteristics of sample at T1

\begin{tabular}{|c|c|c|c|}
\hline & Non-smoker $(n=166)$ & Smoker $(n=27)$ & Total $(N=193)$ \\
\hline Male, $n(\%)$ & $137(83)$ & $19(70)$ & $156(81)$ \\
\hline Age (years), $\mathrm{M} \pm \mathrm{SD}$ (range)*** & $65.23 \pm 9.81$ & $57.44 \pm 11.40$ & $64.14 \pm 10.37(38-91)$ \\
\hline Income $<$ AU\$20 000, $n$ (\%) & $54(33)$ & $9(33)$ & $63(33)$ \\
\hline Married, $n$ (\%) & $131(79)$ & $15(56)$ & $146(76)$ \\
\hline Hypertension, $n$ (\%) & $127(77)$ & $23(85)$ & $150(78)$ \\
\hline Diabetes, $n$ (\%) & $40(24)$ & $5(19)$ & $45(23)$ \\
\hline CABG, $n(\%)^{* *}$ & $104(63)$ & $8(30)$ & $112(58)$ \\
\hline BMI $\left(\mathrm{kg} / \mathrm{m}^{2}\right), \mathrm{M} \pm \mathrm{SD}$ (range) & $28.07 \pm 4.28$ & $27.55 \pm 3.92$ & $28.00 \pm 4.22(20-40)$ \\
\hline LVEF $<30 \% n(\%)$ & $11(7)$ & $0(0)$ & $11(8)$ \\
\hline Alcohol - Excessive & $34(12)$ & $6(22)$ & $40(21)$ \\
\hline Functional status, $\mathrm{M} \pm \mathrm{SD}$ (range) & $24.81 \pm 4.57$ & $23.97 \pm 6.33$ & $24.09 \pm 6.11(0-30)$ \\
\hline$M D^{* * *}$ & $23(14)$ & $12(44)$ & $35(18)$ \\
\hline$A D D^{* * *}$ & $36(33)$ & $18(67)$ & $54(28)$ \\
\hline GAD* & $27(16)$ & $10(37)$ & $37(19)$ \\
\hline History of depression & $57(34)$ & $10(37)$ & $67(35)$ \\
\hline Antidpressants at T1, $n$ (\%) & $17(10)$ & $3(11)$ & $20(11)$ \\
\hline Antidepressants T1-T3, $n$ (\%) & $13(8)$ & $3(11)$ & $16(8)$ \\
\hline Neuroticism, $\mathrm{M} \pm \mathrm{SD}$ (range) ${ }^{* *}$ & $26.48 \pm 8.50$ & $32.44 \pm 10.56$ & $27.32 \pm 9.02(10-50)$ \\
\hline Social support, $\mathrm{M} \pm \mathrm{SD}$ (range) ${ }^{* *}$ & $70.25 \pm 11.19$ & $63.89 \pm 12.34$ & $69.36 \pm 11.54(21-84)$ \\
\hline Attended cardiac rehabilitation ${ }^{\dagger}, n(\%)^{*}$ & $121(76)$ & $13(50)$ & $134(72)$ \\
\hline
\end{tabular}

$A D D$ Any depressive disorder, $B M I$ body mass index, CABG coronary artery bypass graft surgery, GAD Generalized Anxiety Disorder, $L V E F$ left ventricular ejection fraction, $M D D$ Major Depressive Disorder.

${ }^{\dagger}$ Known for $\mathbf{n}=187$.

${ }^{*} P<.05{ }^{* *} P<.01{ }^{* * *} P<.001$. 
Table 2 Direct logistic regression analyses to predict the association between smoking and depression (MDD, 'ADD' and T2 Depression)

\begin{tabular}{|c|c|c|c|c|c|c|c|c|c|c|c|c|c|c|c|c|c|c|}
\hline \multirow[b]{3}{*}{ Variable } & \multicolumn{6}{|c|}{ T1 MDD $(N=193)$} & \multicolumn{6}{|c|}{ T1 'ADD' $(N=193)$} & \multicolumn{6}{|c|}{ T2 Depression $(N=189)$} \\
\hline & \multirow[t]{2}{*}{ B } & \multirow[t]{2}{*}{ SE } & \multirow[t]{2}{*}{ Wald } & \multirow[t]{2}{*}{ OR } & \multicolumn{2}{|l|}{$95 \% \mathrm{Cl}$} & \multirow[b]{2}{*}{ B } & \multirow[b]{2}{*}{ SE } & \multirow[b]{2}{*}{ Wald } & \multirow[b]{2}{*}{ OR } & \multicolumn{2}{|l|}{$95 \% \mathrm{Cl}$} & \multirow[b]{2}{*}{ B } & \multirow[b]{2}{*}{ SE } & \multirow[b]{2}{*}{ Wald } & \multirow[b]{2}{*}{ OR } & \multicolumn{2}{|l|}{$95 \% \mathrm{Cl}$} \\
\hline & & & & & Lower & Upper & & & & & Lower & Lower & & & & & Lower & Upper \\
\hline Male & -.24 & .64 & .14 & .79 & .23 & 2.77 & .19 & .59 & .10 & 1.21 & .38 & 3.83 & .57 & .70 & .65 & 1.76 & .44 & 6.97 \\
\hline Married & .79 & .74 & 1.15 & 2.21 & .52 & 9.43 & .48 & .63 & .596 & 1.62 & .47 & 5.56 & .67 & .74 & .81 & 1.96 & .46 & 8.39 \\
\hline Income & .57 & .61 & .86 & 1.76 & .53 & 5.82 & .12 & .54 & .05 & 1.12 & .39 & 3.24 & -.20 & .65 & .09 & .82 & .23 & 2.96 \\
\hline LVEF & -18.86 & 1.10 & .00 & .00 & .00 & . & -.42 & 1.22 & .12 & .65 & .06 & 7.12 & -18.51 & 1.13 & .00 & .00 & .00 & . \\
\hline BMI & -.04 & .06 & .41 & .96 & .85 & 1.09 & .04 & .05 & .74 & 1.05 & .95 & 1.15 & -.02 & .07 & 1.00 & .98 & .85 & 1.12 \\
\hline Diabetes & -.35 & .66 & .29 & .70 & .19 & 2.54 & -.51 & .58 & .80 & .60 & .19 & 1.85 & -.65 & .71 & .84 & .52 & .13 & 2.09 \\
\hline Smoking & 1.46 & .69 & $4.52^{*}$ & 4.30 & 1.12 & 16.46 & 2.08 & .63 & $11.05^{* *}$ & 8.03 & 2.35 & 27.46 & .16 & .71 & .05 & 1.17 & .29 & 4.69 \\
\hline $\begin{array}{l}\text { Alcohol - } \\
\text { excessive }\end{array}$ & -.70 & .66 & 1.13 & .50 & .14 & 1.81 & -.07 & .55 & .02 & .93 & .32 & 2.71 & .05 & .64 & .01 & 1.05 & .30 & 3.71 \\
\hline $\begin{array}{l}\text { History of } \\
\text { depression }\end{array}$ & .94 & .53 & 3.13 & 2.56 & .90 & 7.23 & .75 & .46 & 2.65 & 2.12 & .86 & 5.22 & .68 & .56 & 1.51 & 1.98 & .67 & 5.88 \\
\hline GAD & 1.87 & .60 & $9.56^{* *}$ & 6.50 & 1.98 & 21.09 & 1.81 & .55 & $10.74^{* *}$ & 6.09 & 2.07 & 17.93 & .05 & .69 & .01 & 1.06 & .27 & 4.11 \\
\hline Neuroticism & .08 & .04 & $4.37^{*}$ & 1.08 & 1.01 & 1.16 & .09 & .03 & $7.67^{* *}$ & 1.09 & 1.03 & 1.16 & .08 & .04 & $4.32^{*}$ & 1.09 & 1.01 & 1.17 \\
\hline $\begin{array}{l}\text { Social } \\
\text { support }\end{array}$ & .01 & .03 & .15 & 1.01 & .96 & 1.06 & .01 & .02 & .20 & 1.01 & .97 & 1.05 & -.06 & .02 & $6.35^{*}$ & .94 & .90 & .99 \\
\hline $\begin{array}{l}\text { Functional } \\
\text { status }\end{array}$ & -.07 & .04 & 2.64 & .93 & .86 & 1.01 & -.08 & .04 & $5.15^{*}$ & .92 & .86 & .99 & -.11 & .05 & $5.76^{*}$ & .90 & .82 & .98 \\
\hline CABG & .91 & .53 & 2.94 & 2.49 & .88 & 7.04 & 1.10 & .45 & $6.07^{*}$ & 3.01 & 1.25 & 7.24 & 1.23 & .57 & $4.64^{*}$ & 3.43 & 1.12 & 10.54 \\
\hline Constant & -3.83 & 3.25 & 1.38 & .02 & & & -5.43 & 2.69 & 4.06 & .00 & & & .96 & 3.06 & 1.00 & 2.62 & & \\
\hline
\end{tabular}

$A D D$ Any depressive disorder, $B M I$ body mass index, CABG Coronary artery bypass graft surgery, GAD Generalized Anxiety Disorder, Income Annual household income $\leq$ AU\$20 000 LVEF left ventricular ejection fraction $<30 \%, M D D$ Major Depressive Disorder.

${ }^{*} P<.05{ }^{*} P<.01 * * * P<.001$.

likelihood of being classified as 'ever depressed' by an OR of 5.19 [95\% CI, 1.51-17.82; $P<.01]$ and was again the most important predictor of depression in this analysis.

\section{Association between smoking and HRQOL}

Correlations between T1 demographic, psychosocial and clinical variables and T3 mental and physical HRQOL are shown in Table 4. Multivariate analyses to investigate the association between smoking and HRQOL at T3 are shown in Table 5. The model to predict MCS explained $47 \%$ of the variance in outcome $[F(14,184)=10.85$, $P<.001]$ and showed that smoking was significantly associated with MCS $(P<.001)$, explaining approximately $4.8 \%$ of unique variance. Neuroticism was the most important predictor and contributed approximately $6.7 \%$ of unique variance. The model to predict PCS explained $46 \%$ of the variance in outcome $[F(14,184)=10.24, P<.001]$. Smoking was not an independent predictor of PCS $(P=.98)$.

\section{Discussion}

Our findings indicate that in the presence of well-known confounders, exposure to smoking is independently associated with a 4.30- and a 8.03-fold increase in odds for MDD and a diagnosis of minor depression, dysthymia or $\mathrm{MDD}$ as a combined group ('ADD'), respectively, 3 months following cardiac hospitalization. Smoking increased the likelihood of being classified as depressed according to study criteria at least once during the 9 months of the study period by an OR of 5.19. Despite this finding, there was no significant association between smoking and depression at T2 or T3, and given that smoking status and depression were measured simultaneously at $\mathrm{T} 1$, an independent prospective association cannot be clearly concluded from these data. This study provides evidence consistent with the hypothesis that tobacco smoking is an independent predictor of depression and adds to the evidence that smoking is noxious to mental health [68]. Consistent with prediction, smoking was associated with worse mental HRQOL. There was, however, no association between smoking and physical HRQOL. These findings are similar to Rumsfeld et al. (2003) [69] who reported that tobacco smoking was independently predictive of 6-month mental but not physical HRQOL in CAD patients who underwent revascularization.

The mechanisms through which smoking is associated with depression are likely to be complex, involving biogenetic, psychological and environmental factors. Indeed, studies in depression have supported a tri-directional relationship driven by mutually reinforcing effects and shared causal factors [70]. These factors include genetic and 
Table 3 Direct logistic regression analyses to predict the association between smoking and depression (T3 Depression, 'Persistently depressed' and 'Ever depressed')

\begin{tabular}{|c|c|c|c|c|c|c|c|c|c|c|c|c|c|c|c|c|c|c|}
\hline \multirow[b]{3}{*}{ Variable } & \multicolumn{6}{|c|}{ T3 Depression $(N=184)$} & \multicolumn{6}{|c|}{ ‘Persistently depressed' $(N=184)$} & \multicolumn{6}{|c|}{ 'Ever depressed' $(N=184)$} \\
\hline & \multirow[t]{2}{*}{ B } & \multirow[t]{2}{*}{ SE } & \multirow[t]{2}{*}{ Wald } & \multirow[t]{2}{*}{ OR } & \multicolumn{2}{|c|}{$95 \% \mathrm{Cl}$} & \multirow[b]{2}{*}{ B } & \multirow[b]{2}{*}{ SE } & \multirow[b]{2}{*}{ Wald } & \multirow[b]{2}{*}{ OR } & \multicolumn{2}{|c|}{$95 \% \mathrm{Cl}$} & \multirow[b]{2}{*}{ B } & \multirow[b]{2}{*}{ SE } & \multirow[b]{2}{*}{ Wald } & \multirow[b]{2}{*}{ OR } & \multicolumn{2}{|l|}{$95 \% \mathrm{Cl}$} \\
\hline & & & & & Lower & Upper & & & & & Lower & Lower & & & & & Lower & Upper \\
\hline Male & .31 & .68 & .21 & 1.37 & .360 & 5.20 & .04 & .82 & .00 & 1.04 & .21 & 5.15 & .82 & .62 & 1.72 & 2.26 & .67 & 7.66 \\
\hline Married & 1.87 & .93 & $4.11^{*}$ & 6.52 & 1.06 & 39.89 & .60 & 1.10 & .31 & 1.82 & .235 & 14.63 & .91 & .68 & 1.79 & 2.48 & .66 & 9.39 \\
\hline Income & -.81 & .73 & 1.23 & .45 & .11 & 1.86 & .01 & .81 & .00 & 1.01 & .21 & 4.94 & .01 & .55 & .00 & 1.01 & .35 & 2.96 \\
\hline LVEF & -18.54 & 1.10 & .00 & .00 & .00 & . & -17.66 & 1.13 & .00 & .00 & .00 & . & -1.08 & 1.19 & .84 & .34 & .03 & 3.45 \\
\hline BMI & .10 & .06 & 3.04 & 1.11 & .99 & 1.25 & -.05 & .09 & .33 & .95 & .80 & 1.13 & .03 & .05 & .47 & 1.03 & .94 & 1.14 \\
\hline Diabetes & 1.11 & .63 & 3.12 & 3.05 & .88 & 10.51 & .15 & .84 & .03 & 1.16 & .23 & 5.99 & -.78 & .58 & 1.83 & .46 & .15 & 1.42 \\
\hline Smoking & .43 & .74 & .34 & 1.54 & .36 & 6.58 & .06 & .91 & .01 & 1.07 & .18 & 6.32 & 1.65 & .63 & $6.84^{* *}$ & 5.19 & 1.51 & 17.82 \\
\hline $\begin{array}{l}\text { Alcohol - } \\
\text { excessive }\end{array}$ & .18 & .72 & .06 & 1.19 & .29 & 4.91 & -.61 & .93 & .43 & .54 & .09 & 3.35 & .34 & .56 & .36 & 1.40 & .47 & 4.23 \\
\hline $\begin{array}{l}\text { History of } \\
\text { depression }\end{array}$ & .62 & .55 & 1.27 & 1.86 & .63 & 5.55 & 1.69 & .77 & $4.87^{*}$ & 5.41 & 1.21 & 24.23 & .15 & .47 & .10 & 1.16 & .46 & 2.93 \\
\hline GAD & 1.38 & .65 & $4.53^{*}$ & 3.99 & 1.12 & 14.28 & 1.04 & .876 & 1.45 & 2.83 & .52 & 15.48 & 1.43 & .56 & $6.45^{*}$ & 4.16 & 1.38 & 12.48 \\
\hline Neuroticism & .06 & .04 & 2.59 & 1.06 & .99 & 1.14 & .04 & .05 & .52 & 1.04 & .94 & 1.14 & .13 & .03 & $14.41^{* * *}$ & 1.14 & 1.06 & 1.21 \\
\hline $\begin{array}{l}\text { Social } \\
\text { support }\end{array}$ & -.07 & .03 & $5.91^{*}$ & .94 & .89 & .99 & -.04 & .03 & 1.77 & .96 & .91 & 1.02 & -.02 & .02 & .62 & .98 & .94 & 1.03 \\
\hline $\begin{array}{l}\text { Functional } \\
\text { status }\end{array}$ & -.13 & .05 & $5.96^{*}$ & .88 & .80 & .98 & -.06 & .06 & .95 & .95 & .84 & 1.058 & -.18 & .05 & $15.52^{* * *}$ & .84 & .77 & .92 \\
\hline CABG & .49 & .56 & .78 & 1.63 & .55 & 4.84 & 1.13 & .72 & 2.51 & 3.11 & .76 & 12.653 & .75 & .44 & 2.88 & 2.11 & .89 & 4.98 \\
\hline Constant & -2.01 & 3.07 & .43 & .13 & & & .04 & .82 & .00 & 1.04 & .21 & 5.153 & -2.15 & 2.56 & .71 & .12 & & \\
\hline
\end{tabular}

$A D D$ Any depressive disorder, $B M I$ body mass index, CABG Coronary artery bypass graft surgery, GAD Generalized Anxiety Disorder, Income Annual household income $\leq$ AU\$20 000, LVEF left ventricular ejection fraction $<30 \%, M D D$ Major Depressive Disorder.

${ }^{*} P<.05{ }^{* *} P<.01 * * * P<.001$.

Table 4 Correlations between T1 demographic, psychosocial and clinical variables and T3 mental and physical HRQOL

\begin{tabular}{|c|c|c|c|c|c|c|c|c|c|c|c|c|c|c|c|c|}
\hline Variable & 1 & 2 & 3 & 4 & 5 & 6 & 7 & 8 & 9 & 10 & 11 & 12 & 13 & 14 & 15 & 16 \\
\hline 1. Male & 1 & & & & & & & & & & & & & & & \\
\hline 2. Married & $.28^{* * *}$ & 1 & & & & & & & & & & & & & & \\
\hline 3. Income & -.14 & $-.27^{* * *}$ & 1 & & & & & & & & & & & & & \\
\hline 4. LVEF & .06 & -.02 & .07 & 1 & & & & & & & & & & & & \\
\hline 5. BMI & -.06 & .09 & -.01 & -.05 & 1 & & & & & & & & & & & \\
\hline 6. Diabetes & -.04 & -.06 & $.17^{*}$ & .02 & .10 & 1 & & & & & & & & & & \\
\hline 7. Smoking & -.11 & $-.19^{* * *}$ & .01 & -.10 & -.04 & -.05 & 1 & & & & & & & & & \\
\hline 8. Alcohol -excessive & $.18^{*}$ & .08 & -.06 & -.07 & -.07 & -.13 & .02 & 1 & & & & & & & & \\
\hline 9. History ofdepression & -.06 & -.09 & .05 & -.09 & .09 & -.04 & .02 & .00 & 1 & & & & & & & \\
\hline 10. GAD & -.03 & .00 & .05 & -.12 & .04 & -.08 & $.18^{*}$ & .01 & $.23^{* * *}$ & 1 & & & & & & \\
\hline 11. Neuroticism & -.10 & .03 & $.15^{*}$ & -.11 & .04 & .03 & $.23^{* * *}$ & .00 & $.34^{* * *}$ & $.49^{* *}$ & 1 & & & & & \\
\hline 12. Social support & .06 & $.34^{* * *}$ & -.10 & .04 & .00 & .04 & $-.20^{* * *}$ & .05 & -.08 & $-.19 * *$ & -.12 & 1 & & & & \\
\hline 13. Functional status & $20 * * *$ & $.19^{* * *}$ & $-.42^{* * *}$ & -.07 & -.07 & $-.20^{* * *}$ & .02 & .11 & $-.16^{*}$ & -.06 & $-.17^{*}$ & .11 & 1 & & & \\
\hline 14. CABG & -.12 & -.06 & 0.08 & -.03 & .00 & -.10 & $.23^{* *}$ & .06 & -.03 & .09 & .13 & .00 & .01 & 1 & $-.15^{*}$ & \\
\hline 15. MCS & .100 & .02 & -.06 & .09 & $-.26^{* * *}$ & -.08 & $-.36^{* * *}$ & .07 & $-.32^{* * *}$ & $-.41^{* * *}$ & $-.54^{* * *}$ & $.22^{* * *}$ & $.21^{* * *}$ & & 1 & \\
\hline 16. PCS & $.16^{*}$ & $.16^{*}$ & $-.29 * * *$ & -.01 & -.07 & $-.23^{* * *}$ & -.04 & .06 & $-.28^{* * *}$ & .00 & $-.21^{* * *}$ & .06 & $.61^{* * *}$ & $.18^{* * *}$ & & 1 \\
\hline
\end{tabular}

BMI body mass index, CABG Coronary artery bypass graft surgery, GAD Generalized Anxiety Disorder, Income Annual household income $\leq$ AU $\$ 20000$, LVEF left ventricular ejection fraction $<30 \%$, MCS Mental Component Summary score of the SF-36, PCS Physical Component Summary score of the SF-36. ${ }^{*} P<.05 * * P<.01 * * * P<.001$. 
Table 5 Multiple regression analyses to predict the association between smoking and HRQOL at T3

\begin{tabular}{|c|c|c|c|c|c|c|c|c|c|c|}
\hline \multirow{3}{*}{ Variable } & \multicolumn{5}{|c|}{ Physical HRQOL at T3 $(N=184)$} & \multicolumn{5}{|c|}{ Mental HRQOL at T3 $(N=184)$} \\
\hline & \multirow[b]{2}{*}{ B } & \multirow[b]{2}{*}{ SE } & \multirow[b]{2}{*}{$\beta$} & \multicolumn{2}{|c|}{$95 \% \mathrm{Cl}$ for $\beta$} & \multirow[b]{2}{*}{ B } & \multirow[b]{2}{*}{ SE } & \multirow[b]{2}{*}{$\beta$} & \multicolumn{2}{|c|}{$95 \% \mathrm{Cl}$ for $\beta$} \\
\hline & & & & Lower & Upper & & & & Lower & Upper \\
\hline Constant & 2191.21 & 471.57 & & 1260.29 & 3122.14 & 5094.85 & 530.41 & & 4047.77 & 6141.92 \\
\hline Male & -1.29 & 134.42 & .00 & -266.64 & 264.06 & -78.07 & 151.19 & -.03 & -376.53 & 220.39 \\
\hline Married & 82.47 & 135.80 & .04 & -185.61 & 350.56 & -77.48 & 152.75 & -.03 & -379.01 & 224.06 \\
\hline Income & -3.39 & 120.15 & -.00 & -240.57 & 233.80 & 151.72 & 135.14 & .07 & -115.06 & 418.50 \\
\hline LVEF & 67.40 & 212.32 & .02 & -351.75 & 486.55 & -47.46 & 238.81 & -.01 & -518.90 & 423.99 \\
\hline BMI & 2.54 & 11.90 & .01 & -20.95 & 26.02 & -50.37 & 13.38 & $-.22^{* * *}$ & -76.78 & -23.95 \\
\hline Diabetes & -240.51 & 120.41 & $-.12^{*}$ & -478.22 & -2.81 & -148.65 & 135.44 & -.07 & -416.01 & 118.72 \\
\hline Smoking & -3.08 & 154.25 & -.00 & -307.58 & 301.42 & -687.49 & 173.49 & $-.24^{* * *}$ & -1029.98 & -345.01 \\
\hline Alcohol - excessive & -15.11 & 127.08 & -.01 & -265.97 & 235.75 & 125.54 & 142.93 & .05 & -156.62 & 407.69 \\
\hline History of depression & -325.52 & 114.85 & $-.18^{* *}$ & -552.24 & -98.79 & -252.56 & 129.18 & -.12 & -507.57 & 2.45 \\
\hline GAD & 249.36 & 146.24 & .11 & -39.33 & 538.04 & -378.52 & 164.48 & $-.15^{*}$ & -703.22 & -53.82 \\
\hline Neuroticism & -10.02 & 6.79 & -.10 & -23.42 & 3.38 & -35.86 & 7.64 & $-.33^{* * *}$ & -50.93 & -20.79 \\
\hline Social support & -.02 & .04 & -.03 & -.09 & .054 & .06 & .04 & .09 & -.02 & .15 \\
\hline Functional status & .06 & .01 & $.55^{* * *}$ & .05 & .074 & .02 & .01 & .12 & -.00 & .03 \\
\hline CABG & -248.66 & 104.18 & -.14 & -454.33 & -43.00 & -106.99 & 117.18 & -.05 & -338.31 & 124.33 \\
\hline
\end{tabular}

$B M I$ body mass index, CABG Coronary artery bypass graft surgery, GAD Generalized Anxiety Disorder, HRQOL Health related quality of life, Income Annual household income $\leq$ AU\$20 000, LVEF left ventricular ejection fraction $<30 \%$. ${ }^{*} P<.05{ }^{* *} P<.01{ }^{* * *} P<.001$.

neural connectivity variables that are common to both depression and smoking; smoking-induced neurobiological changes that might predispose to depression; the transient alleviation of depressive symptoms and psychotropic side effects with smoking; and increased smoking as part of an agitated mental state.

Homeostatic compensation ensures that over time and with adaptation, agents that initially induce short-term alterations in mood later tend to produce contrary effects. For example, the acute effects of alcohol (i.e., anxiolysis and sleep induction) are characteristically the converse of the withdrawal pattern (i.e., anxiety and insomnia). Cigarette smoking can be conceptualised as a chronic dysphoric withdrawal state punctuated by brief reinforcing intoxications. Smoking addiction, in common with other addictions, is mediated via the dopaminergic reward pathway, and dopamine has a key role in depression [71]. Smoking dysregulates the striatal D2 receptor, which may play a shared role in both its pathway to addiction and its effect on mood [72].

The status of both tobacco smoking and depression as risk factors for cardiac morbidity and mortality is supported by evidence of shared pathways. For instance, cigarette smoke contains high amounts of oxidative free radicals, including nitric oxide [73]. These free radicals have a complex effect on the oxidative defence system, inducing a direct oxidative attack and simultaneously upregulating antioxidant defences [73]. Oxidative stress is implicated in the pathophysiology of both depression
[74] and cardiovascular disease [75]. Cigarette smoking has also been associated with raised levels of C-reactive protein [76], suggesting the stimulation of a chronic inflammatory state, which again, is described in both depression and cardiovascular disorders $[77,78]$.

Strengths of the study include its prospective design, the low attrition rate and the inclusion of well-known confounders of depression such as history of depression, neuroticism, anxiety and functional status. We acknowledge that the generalizability of our findings is limited by sample bias. Since respondents were more likely to be male and were on average younger than non-respondents, these findings should be cautiously applied to older, female CAD patients. Indeed, there is evidence that women and men with CAD have different medical, functional and psychosocial profiles [80-82]. Also, taking into account all patients treated during the study period, regardless of eligibility for the study, the compliance rate was low (193 of $528=37 \%$ ). It is possible that patients with depression were less likely to participate in the study potentially resulting in an underestimation of the effect of smoking. Duration and exposure of smoking prior to depression onset are unknown. Baseline measures were not conducted prior to the cardiac interventions. Exact depression status was not known at the time of index cardiac event for the reasons explained earlier, but prior history of depression, the most important risk factor for subsequent depression, was included in all multivariate analyses. Still, it is plausible that depression led to tobacco use and that smokers 
had more depressive symptoms before their cardiac events. It is noted that LVEF can be an imprecise measure of disease severity but was the most consistently available measure in the medical records. We acknowledge that although ORs were significant and notable, the confidence intervals were wide. This has implications of imprecision in estimating the true effect size and may be addressed in future studies with larger sample sizes.

Individuals often need risk to be personalized before a health warning is internalized to the point that it precipitates a decision to act. The message that smoking is an independent predictor of depression in someone who experiences the distress of depression, may be a catalyst for change. This information may work in parallel with the notion that the diagnosis of a smoking-related physical illness can precipitate motivation for smoking discontinuation. The demonstration of the association between smoking and mood and HRQOL augments the well-established physical imperative to encourage smoking cessation in patients with CAD [79]. This message similarly needs to be routinely communicated to depressed individuals.

\section{Conclusions}

Adjusted for well-known confounders of depression, smoking was independently associated with depression in patients with heart disease. Smoking was an independently associated with poor mental HRQOL. These data reinforce the imperative to encourage smoking cessation in patients with heart disease, and add to the evidence for smoking cessation campaigns in the primary prevention of depression [8].

\footnotetext{
Abbreviations

ADD: Any depressive disorder; BMI: Body mass index; CABG: Coronary artery bypass graft surgery; CAD: Coronary artery disease; CIDI: Composite International Diagnostic Interview; DSM-IV: Diagnostic and Statistical Manual Version 4; GAD: Generalized Anxiety Disorder; HADS: Hospital Anxiety Depression Scale; HRQOL: Health related quality of life; LVEF: Left ventricular ejection fraction; MCS: Mental functioning aggregate summary score of the SF-36; MI: Myocardial infarction; MDD: Major depressive disorder; MSPSS: Multidimensional Scale of Perceived Social Support; MINI: Mini International Neuropsychiatric Interview Version 5; PCS: Physical functioning aggregate summary score of the SF-36; PTCA: Percutaneous transluminal coronary angioplasty; SCID: Structured Clinical Interview for DSM-IV; SF-36: Short Form-36.
}

\section{Competing interests}

The authors declare that they have no competing interests.

\section{Authors' contributions}

LS collected and analysed the data. LS, MB, HJ and LS participated in the conception and design of the study. LS wrote the initial draft of the manuscript, $\mathrm{MB}$ and $\mathrm{HJ}$ participated in further manuscript writing. All authors read and approved the final manuscript.

\section{Acknowledgements}

Dr Lesley Stafford wishes to thank the University of Melbourne for their financial contribution towards the study. The authors thank Dr Sandy Black and Dr Morteza Mohajeri of the Geelong Hospital for facilitating the project.

\section{Disclosures}

Lesley Stafford has no disclosures.

Henry Jackson receives royalties for books and book chapters from McGraw Hill and Cambridge University Press.

Michael Berk has received Grant/Research Support from the NIH, Cooperative Research Centre, Simons Autism Foundation, Cancer Council of Victoria, Stanley Medical Research Foundation, MBF, NHMRC, Beyond Blue, Rotary Health, Geelong Medical Research Foundation, Bristol Myers Squibb, Eli Lilly, Glaxo SmithKline, Organon, Novartis, Mayne Pharma and Servier, has been a speaker for Astra Zeneca, Bristol Myers Squibb, Eli Lilly, Glaxo SmithKline, Janssen Cilag, Lundbeck, Merck, Pfizer, Sanofi Synthelabo, Servier, Solvay and Wyeth, and served as a consultant to Astra Zeneca, Bristol Myers Squibb, Eli Lilly, Glaxo SmithKline, Janssen Cilag, Lundbeck Merck and Servier.

\section{Author details}

${ }^{1}$ Centre for Women's Mental Health, Royal Women's Hospital, Parkville, Australia. ${ }^{2}$ Melbourne School of Psychological Sciences, University of Melbourne, Melbourne, Australia. ${ }^{3}$ School of Medicine, Deakin University, Deakin, Australia. ${ }^{4}$ ORYGEN Youth Health, Parkville, Australia. ${ }^{5}$ Mental Health Research Institute, Parkville, Australia. ${ }^{6}$ Department of Psychiatry, University of Melbourne, Melbourne, Australia.

Received: 16 August 2012 Accepted: 3 May 2013

Published: 24 May 2013

\section{References}

1. Ridolfo B, Stevenson C. The quantification of drug-caused mortality and morbidity in Australia, 1998. Canberra: AlHW; 2001. Report No.: AlHW cat. no. PHE 29 (Drug Statistics Series no. 7). Available from: http://www.aihw.gov. au/WorkArea/DownloadAsset.aspx?id=6442459309.

2. Australian Institute of Health and Welfare: Australia's health 2006. AlHW cat. no. AUS 73. Canberra: AlHW. Canberra; 2006.

3. Vanable PA, Carey MP, Carey KB, Maisto SA: Smoking among psychiatric outpatients: relationship to substance use, diagnosis, and illness severity. Psychol Addict Behav 2003, 17(4):259-265.

4. Chandra PS, Carey MP, Jairam KR, Girish NM, Rudresh HP: Prevalence and correlates of tobacco use and nicotine dependence among psychiatric patients in India. Addict Behav 2005, 30:1290-1299.

5. Grant BF, Hasin DS, Chou SP, Stinson FS, Dawson DA: Nicotine dependence and psychiatric disorders in the United States: results from the national epidemiologic survey on alcohol and related conditions. Arch Gen Psychiatry 2004, 61:1107-1115.

6. Diwan A, Castine M, Pomerlau CS, Meador-Woodruff JH, Dalack GW: Differential prevalence of cigarette smoking in patients with schizophrenia vs mood disorders. Schizophr Res 1998, 33:113-118.

7. Berk M, Ng F, Wang WV, Tohen M, Lubman DI, Vieta E, Dodd S: Going up in smoke: Tobacco smoking is associated with worse treatment outcomes in mania. J Affect Disord 2008, 110:126-134.

8. Berk M: Should we be targeting smoking as a routine intervention? Acta Neuropsychiatrica 2007, 19:131-132.

9. Vanable PA, Carey MP, Carey KB, Maisto SA: Smoking among psychiatric outpatients: relationship to substance use, diagnosis, and illness severity. Psychol Addict Behav 2003, 17:259-265.

10. Nunes S, Vargas H, Brum J, Prado E, Vargas M, de Castro M, Dodd S, Berk M: A comparison of inflammatory markers in depressed and nondepressed smokers. Nicotine Tob Res 2012, 14(5):540-546.

11. Breslau N, Peterson E, Schultz L, Chilcoat H, Andreski P: Major depression and stages of smoking: A longitudinal investigation. Arch Gen Psychiatry 1998, 55:151-156.

12. Kendler KS, Neale MC, maclean CJ, Heath AC, Eaves LJ, Kessler RC: Smoking and major depression: a causal analysis. Arch Gen Psychiatry 1993, 50:36-43.

13. Pasco JA, Williams LJ, Jacka FN, Ng F, Henry MJ, Nicholson GC, Kotowicz MA: Tobacco smoking as a risk factor for major depressive disorder:a population-based study. Br J Psychiatry 2008, 193:322-6.

14. Nicholson A, Kuper $H$, Hemingway $H$ : Depression as an aetiologic and prognostic factor in coronary heart disease: A meta-analysis of 6362 events among 146538 participants in 54 observational studies. Eur Heart J 2006, 27(23):2763-2774.

15. Kuper $\mathrm{H}$, Marmot $M$, Hemingway $H$ : Systematic review of prospective cohort studies of psychosocial factors in the etiology and prognosis of coronary heart disease. Sem Vasc Med 2002, 2(3):267-314. 
16. Van Melle J, de Jonge P, Spijkerman TA, Tijssen J, Ormel J, van Veldhuisen D, Van Den Brink RHS, Van Den Berg MP: Prognostic association of depression following myocardial infarction with mortality and cardiovascular events: A meta-analysis. Psychosom Med 2004, 88:814-822.

17. Waters D, Lesperance F, Gladstone P, Boccuzzi S, Cook T, Hudgin R, Krip G, Higginson L: Effects of cigarette smoking on the angiographic evoluation of coronary atherosclerosis: A Canadian Coronary Atherosclerosis Intervention Trial (CCAIT) Substudy. Circulation 1996, 94:614-621.

18. Gabbay FH, Krantz DS, Kop WJ, Hedges SM, Klein J, Gottdiener JS, Rozanski A: Triggers of myocardial ischemia during daily life in patients with coronary artery disease: Physical and mental activities, anger and smoking. J Am Col Cardiol 1996, 27:585-592.

19. Lane D, Carroll D, Ring C, Beevers D, Lip GYH: Mortality and quality of life 12 months after myocardial infarction: Effects of depression and anxiety. Psychosom Med 2001, 63(2):221-230.

20. Lesperance F, Frasure-Smith $\mathrm{N}$ : Negative emotions and coronary heart disease: Getting to the heart of the matter. Lancet 1996, 347(8999):414-415.

21. Sorensen C, Brandes A, Hendricks O, Thrane J, Friis-Hasche E, Haghfelt T, Bech P: Psychosocial predictors of depression in patients with acute coronary syndrome. Acta Psychiatr Scand 2005, 111(2):116-124.

22. Schrader G, Cheok F, Hordacre A, Guiver N: Predictors of depression three months after cardiac hospitalization. Psychosom Med 2004, 66:514-520.

23. Schrader G, Cheok F, Hordacre A-L, Marker J: Predictors of depression 12 months after cardiac hospitalization: The Identifying Depression as a Comorbid Condition study. A N ZJ Psychiatry 2006, 40(11-12):1025-1030.

24. Lyons RS, Lo SV, Littlepage BNC: Perception of health among ever-smokers and never-smokers: a comparison using the SF-36 Health Survey Questionnaire. Tob Control 1994, 2:213-215.

25. Tillman M, Silcock J: A comparison of smokers' and ex-smokers' health-related quality of life. J Public Health Med 1997, 19:268-273.

26. Hirdes JP, Maxwell CJ: Smoking cessation and quality of life outcomes among older adults in teh Campbell's survey on well-being. Can J Public Health 1994, 85:99-102.

27. Taira DA, Seto TB, Ho KK, Krumholz HM, Cutlip DE, Berezin R, Kuntz RE, Cohen DJ: Impact of smoking on health-related quality of life after percutaneous coronary revascularization. Circulation 2000, 102:1369-1374.

28. Henry M, Pasco J, Seeman E, Nicholson G, Kotowicz M: Prevalence of osteoporosis in Australian women: Geelong Osteoporosis Study. J Clinl Densitom 2000, 3:261-268.

29. Barth J, Schumaker M, Herrmann-Lingen C: Depression as a risk factor for mortality in patients with coronary heart disease: A meta-analysis. Psychosom Med 2004, 66:802-813.

30. Burg MM, Benedetto C, Rosenberg R, Soufer R: Presurgical depression predicts medical morbidity 6 months after coronary artery bypass graft surgery. Psychosom Med 2005, 65(1):111-118.

31. Sullivan MD, LaCroix AZ, Russo JE, Walker EA: Depression and self-reported physical health in patients with coronary disease: Mediating and moderating factors. Psychosom Med 2001, 63(2):248-256

32. Kaptein $\mathrm{Kl}$, De Jonge $\mathrm{P}$, Van den Brink RHS, Korf J: Course of depressive symptoms after myocardial infarction and cardiac prognosis: A latent class analysis. Psychosom Med 2006, 68(5):662-668.

33. Potts MK, Daniels M, Burnam MA, Wells KB: A structured interview version of the Hamilton Depression Rating Scale: evidence of reliability and versatility of administration. J Psychiatr Res 1990, 24(4):335-350.

34. Simon GE, Revicki D, VonKorff M: Telephone assessment of depression severity. J Psychiatr Res 1993, 27(3):247-252.

35. National Heart Foundation of Australia and Cardiac Society of Australia and New Zealand: Reducing risk in heart disease: Guidelines for preventing cardiovascular events in people with coronary heart disease. Melbourne, Victoria: National Heart Foundation of Australia; 2004

36. Sheehan DV, Lecrubier Y, Sheehan KH, Janavs J, Weiller E, Keskiner A Schinka J, Knapp E, Sheehan MF, Dunbar GC: The validity of the Mini International Neuropsychiatric Interview (M.I.N.I) according to the SCID-P and its reliability. Eur Psychiatry 1997, 12:232-241.

37. Lecrubier $Y$, Sheehan DV, Weiller E, Amorim P, Bonora I, Sheehan KH, Janavs J, Dunbar GC: The Mini International Neuropsychiatric Interview (M.I.N.I.). A short diagnostic structured interview: Reliability and validity according to the CIDI. Eur Psychiatry 1997, 12:224-231.

38. First M, Spitzer R, Gibbon M, Williams B: Structured Clinical Interview for DSM-IV TR Axis I Disorders, Research Version, Non-Patient Edition. New York: Biometrics Research, New York State Psychiatric Institute; 2002.
39. Spitzer RL, Williams JBW, Gibbon M, First MB: Structured Clinical Interview for DSM-III-R. Washington, DC: American Psychiatric Press; 1990.

40. Organization WH: The Composite International Diagnostic Interview (CIDI) Version 1.0. Geneva: World Health Organization; 1990.

41. Sheehan DV, Lecrubier $Y$, Sheehan KH, Amorim P, Janavs J, Weiller $E_{1}$ Hergueta T, Baker R, Dunbar GC: The Mini-International Neuropsychiatric Interview (M.I.N.I): The development and validation of a structured diagnostic psychiatric interview for DSM-IV and ICD-10. J Clin Psychiatry 1998, 59(S20):22-33.

42. Zigmond AS, Snaith RP: The Hospital Anxiety and Depression Scale. Acta Psychiatr Scand 1983, 67:361-370.

43. Herrmann C: International experiences with the Hospital Anxiety and Depression Scale: A review of validation data and clinical results. J Psychosom Res 1997, 42(1):17-41.

44. Bjelland I, Dahl AA, Haug TT, Neckelmann D: The validity of the Hospital Anxiety and Depression Scale: An updated literature review. J Psychosom Res 2002, 52:69-77.

45. Doyle F, McGee HM, De La Harpe D, Shelley E, Conroy R: The Hospital Anxiety and Depression Scale depression subscale, but not the Beck Depression Inventory-Fast Scale, identifies patients with acute coronary syndrome at elevated risk of 1-year mortality. J Psychosom Res 2006, 60(5):461-467.

46. Lewin B, Robertson I, Cay E, Irving J, Campbell M: Effects of self-help post-myocardial infarction rehabilitation on psychological adjustment and use of health services. Lancet 1992, 39(8800):1036-1040.

47. Mayou R, Gill D, Thompson DR, Day A, Hicks N, Volmink J, Neil A: Depression and anxiety as predictors of outcome after myocardial infarction. Psychosom Med 2000, 62(2):212-219.

48. Roberts SB, Bonnici DM, Mackinnon AJ, Worcester MC: Psychometric evaluation of the Hospital Anxiety and Depression Scale (HADS) among female cardiac patients. Br Jf Health Psychol 2001, 6(4):373-383.

49. Cheok F, Schrader G, Banham D, Marker J, Hordacre AL: Identification, course, and treatment of depression after admission for a cardiac condition: Rationale and patient characteristics for the Identifying Depression as a Comorbid Condition (IDACC) project. Am Heart J 2003, 146(6):978-984.

50. Strik JJMH, Honig A, Lousberg R, Denollet J: Sensitivity and specificity of observer and self-report questionnaires in major and minor depression following myocardial infarction. Psychosomatics 2001, 42:423-428.

51. Zimet GD, Powell SS, Farley GK, Werkman S, Berkoff KA: Psychometric characteristics of the Multidimensional Scale of Perceived Social Support. J Pers Assess 1990, 55(3-4):610-617.

52. Dahlem NW, Zimet GD, Walker RR: The Multidimensional Scale of Perceived Social Support: A confirmation study. J Clin Psychol 1991, 47(6):756-761.

53. Canty-Mitchell J, Zimet GD: Psychometric properties of the Multidimensional Scale of Perceived Social Support. Am J Comm Psychol 2000, 28(3):391-400.

54. Frasure-Smith N, Lesperance F, Gravel G, Masson A, Juneau M, Talajic M, Bourassa MG: Social support, depression and mortality during the first year after myocardial infarction. Circulation 2000, 101(16):1919-1924.

55. Parker G, Heruc G, Hilton T, Olley A, Brotchie H, Hadzi-Pavlovic D, Owen C, Friend C, Walsh WF: Explicating links between acute coronary syndrome and depression: Study design and methods. ANZJ Psychiatry 2006, 40(3):245-252.

56. Oxman TE, Freeman DHJ, Manheimer ED: Lack of social participation or religious strength and comfort as risk factors for death after cardiac surgery in the elderly. Psychosom Med 1995, 57:5-15.

57. Zimet GD, Dahlem NW, Zimet SG, Farley GK: The Multidimensional Scale of Perceived Social Support. J Pers Assess 1988, 52(1):30-41.

58. Costa PTJ: McCrae RR: Revised NEO Personality Inventory (NEO-PI-R) and NEO Five-Factor Inventory (NEO-FFI): Professional manual. Odessa, Florida: Psychological Assessment Resources; 1992

59. Goldberg L: A broad-bandwidth, public-domain personality inventory measuring the lower-level facets of several five-factor models. In Personality Psychology in Europe. Volume 7. Edited by Mervielde I, Deary I, De Fruyt F, Ostendorf F. Tilburg: Tilburg University Press; 1999.

60. International Personality Item Pool. http://ipip.ori.org/.

61. Eysenck $S$, Eysenck $H$, Barrett $P$ : A revised version of the Psychoticism scale. Personality and Individual Differences 1985, 6:21-29.

62. Gow AJ, Whiteman MC, Pattie A, Deary IJ: Goldberg's 'IPIP' Big-Five factor markers: Internal consistency and concurrent validation in Scotland. Personality and Individual Differences 2005, 39:317-329. 
63. Buchanan T, Johnson J, Goldberg L: Implementing a five-factor personality inventory for use on the internet. Eur J Psychol Assess 2005, 21(2):115-127.

64. Ware JE, Snow KK, Kosinski M, Gandek B: SF-36 Health Survey: Manual and Interpretation Guide. Boston: The Health Institute, New England Medical Centre; 1993.

65. Dempster M, Donnelly M: Measuring the health related quality of life of people with ischaemic heart disease. Heart 2000, 83(6):641-644.

66. Campeau L: The Canadian Cardiovascular Society grading of angina pectoris revisited 30 years later. Can J Cardiol 2002, 18(4):439-442.

67. Dolgin M: Nomenclature and Criteria for the Diagnosis of Diseases of the Heart and Great Vessels, 9th edn. Boston: Little, Brown \& Co; 1994.

68. Berk M, Ng F, Wang W, Tohen M, Lubman DI, Vieta E, Dodd S: Going up in smoke: Tobacco smoking is associated with worse treatment outcomes in mania. J Affect Disord 2008, 110:126-34.

69. Rumsfeld JS, Magid DJ, Plomondon ME, Sacks J, Henderson WG, Hlatky MA Sethi GK, Morrison DA, Department of Veterans Affairs with Extremely Serious Operative Mortality (AWESOME) Investigators: Health-related quality of life after percutaneous coronary intervention versus coronary bypass surgery in high-risk patients with medically refractory ischemia. J Am Coll Cardiol 2003, 41(10):1732-1738.

70. Freedland KE, Carney RM, Skala JA: Depression and smoking in coronary heart disease. PsychosomMed 2005, 67:S42-46.

71. Malhi GS, Berk M: Does dopamine dysfunction drive depression? Acta Psychiatr Scand Suppl 2007, 433:116-124.

72. Fehr C, Yakushev I, Hohmann N, Buchholz H-G, Landvogt C, Deckers H, Eberhardt A, Klager M, Smolka MN, Scheurich A, et al: Association of low striatal dopamine D2 receptor availability with nicotine dependence similar to that seen with other drugs of abuse. Am J Psychiatry 2008, 165(4):507-514.

73. Eiserich JP, van der Vliet A, Handelman GJ, Halliwell B, Cross CE: Dietary antioxidants and cigarette smoke-induced biomolecular damage: a complex interaction. Am J Clin Nutr 1995, 62 (6 Suppl):1490S-1500S.

74. Berk M, Ng F, Dean O, Dodd S, Bush Al: Glutathione: a novel treatment target in psychiatry. Trends Pharmacol Sci 2008, 29:346-351.

75. Berk BC: Novel approaches to treat oxidative stress and cardiovascular diseases. Trans Am Clin Climatol Assoc 2007, 118:209-214.

76. Das I: Raised C-reactive protein levels in serum from smokers. Clin Chim Acta 1985, 153(1):9-13.

77. Pasco JA, Nicholson GC, Ng F, Henry MJ, Williams LJ, Kotowicz MA, Hodge JP, Dodd S, Kapczinski F, Gama C, et al: Oxidative stress may be a common mechanism linking major depression and osteoporosis. Acta Neuropsychiatrica 2008, 20:112-6.

78. Berk M, Wadee AA, Kuschke RH, O'Neill-Kerr A: Acute phase proteins in major deperssion. J Psychosom Res 1997, 43:529-534.

79. Fagerstrom K: The epidemiology of smoking: health consequences and benefits of cessation. Drugs 2002, 62(Suppl 2):1-9.

doi:10.1186/1471-2261-13-35

Cite this article as: Stafford et al:: Tobacco smoking predicts depression and poorer quality of life in heart disease. BMC Cardiovascular Disorders 2013 13:35.

\section{Submit your next manuscript to BioMed Central and take full advantage of:}

- Convenient online submission

- Thorough peer review

- No space constraints or color figure charges

- Immediate publication on acceptance

- Inclusion in PubMed, CAS, Scopus and Google Scholar

- Research which is freely available for redistribution 